\title{
NORM ESTIMATES FOR FUNCTIONS OF TWO NON-COMMUTING MATRICES*
}

\author{
MICHAEL GIL ${ }^{\dagger}$
}

\begin{abstract}
A class of matrix valued analytic functions of two non-commuting matrices is considered. A sharp norm estimate is established. Applications to matrix and differential equations are also discussed.
\end{abstract}

Key words. Functions of non-commuting matrices, Norm estimate, Matrix equation, Differential equation.

AMS subject classifications. 15A54, 15A45, 15A60.

1. Introduction and statement of the main result. In the book [5], I.M. Gel'fand and G.E. Shilov have established an estimate for the norm of a regular matrix-valued function in connection with their investigations of partial differential equations. However, that estimate is not sharp; it is not attained for any matrix. The problem of obtaining a sharp estimate for the norm of a matrix-valued function has been repeatedly discussed in the literature, cf. [2]. In the paper [6] (see also [7]), the author has derived an estimate for regular matrix-valued functions, which is attained in the case of normal matrices. In [8], the results of the paper [6] were generalized to functions of two commuting matrices. In the present paper, we establish a sharp estimate for the norm of a matrix-valued function of two non-commuting matrices.

It should be noted that functions of many operators were investigated by many mathematicians, (cf. $[1,15,16]$ and references therein) however the norm estimates were not considered, but as it is well-known, matrix valued functions give us representations of solutions of various differential, difference equations and matrix equations. This fact allows us to investigate stability, well-posedness and perturbations of these equations by norm estimates for matrix valued functions, cf. [2].

Let $\mathbb{C}^{n}$ be the Euclidean space with scalar product $(\cdot, \cdot)$, the Euclidean norm $\|\cdot\|=\sqrt{(\cdot, \cdot)}$ and the unit operator $I$. Unless otherwise stated $A, K$ and $\tilde{A}$ will be $n \times n$ matrices. $\|A\|=\sup _{h \in \mathbb{C}^{n}}\|A h\| /\|h\|$ is the spectral (operator) norm of $A$. By $\sigma(A)$ and $R_{z}(A)=(A-z I)^{-1}(z \notin \sigma(A))$ we denote the spectrum and resolvent of $A$, respectively.

* Received by the editors on December 23, 2010. Accepted for publication on May 14, 2011. Handling Editor: Harm Bart.

${ }^{\dagger}$ Department of Mathematics, Ben Gurion University of the Negev, PO Box 653, Beer-Sheva 84105, Israel (gilmi@bezeqint.net). 
Let $\Omega_{A}$ and $\Omega_{\tilde{A}}$ be open simple connected supersets of $\sigma(A)$ and $\sigma(\tilde{A})$, respectively, and $f$ be a scalar function analytic on $\Omega_{A} \times \Omega_{\tilde{A}}$. We define the matrix valued function

$$
F(f, A, K, \tilde{A}):=-\frac{1}{4 \pi^{2}} \int_{C_{\tilde{A}}} \int_{C_{A}} f(z, w) R_{z}(A) K R_{w}(\tilde{A}) d w d z,
$$

where $C_{A} \subset \Omega_{A}, C_{\tilde{A}} \subset \Omega_{\tilde{A}}$ are closed contour surrounding $\sigma(A)$ and $\sigma(\tilde{A})$, respectively. Such functions play an essential role in the theory of matrix equations. More specifically, consider the matrix equation

$$
\sum_{j=0}^{m_{1}} \sum_{k=0}^{m_{2}} c_{j k} A^{j} X \tilde{A}^{k}=K
$$

where $X$ should be found and $c_{j k}$ are complex numbers. Put

$$
p(z, w)=\sum_{j=0}^{m_{1}} \sum_{k=0}^{m_{2}} c_{j k} z^{j} \tilde{w}^{k}
$$

Then by Theorem 3.1 from [2, Chapter 1] a unique solution of equation (1.2) is given by the formula

$$
X=F\left(\frac{1}{p(z, w)}, A, K, \tilde{A}\right)
$$

provided $\lambda_{k} \neq \tilde{\lambda}_{j}(j, k=1, \ldots, n)$. Throughout the rest of this paper $\lambda_{k}$ and $\tilde{\lambda}_{j}$ are the eigenvalues counted with their multiplicities of $A$ and $\tilde{A}$, respectively. Equations of the type (1.2) naturally arose in various applications, cf. [2, 14, 12]. The Lyapunov equation $A^{*} X+X A=K$, cf. [2], and the Lyapunov type equation

$$
X+A^{*} X A=K
$$

which play an important role in the theory of difference equations, cf. [9] are the examples of equation (1.2). These equations recently attracted the attention of many mathematicians. Mainly, numerical methods for the solutions of matrix equations were developed, cf. [11, 13, 17]. In the paper [3], reflexive and anti-reflexive solutions of a linear matrix equation were explored. No estimates were established for solutions of these equations. Furthermore, suppose that

$$
T(t):=-\frac{1}{4 \pi^{2}} \int_{C_{\tilde{A}}} \int_{C_{A}} e^{t(z+w)} R_{z}(A) K R_{w}(\tilde{A}) d w d z .
$$

Take into account that $z R_{z}(A)=A R_{z}(A)-I$. Then simple calculations show that

$$
T^{\prime}(t)=-\frac{1}{4 \pi^{2}} \int_{C_{\tilde{A}}} \int_{C_{A}}(z+w) e^{t(z+w)} R_{z}(A) K R_{w}(\tilde{A}) d w d z=
$$




$$
-\frac{1}{4 \pi^{2}} \int_{C_{\tilde{A}}} \int_{C_{A}} e^{t(z+w)}\left[A R_{z}(A) K R_{w}(\tilde{A})+R_{z}(A) K R_{w}(\tilde{A}) \tilde{A}\right] d w d z .
$$

So

$$
T^{\prime}(t)=A T(t)+T(t) \tilde{A} .
$$

Such equations arise in numerous applications, in particular in the theory of vector differential equations, cf. [10, p. 509], [2, Section VI.4, equation (4.15) and Section VI.2], [4, Section XV.10]. Additional examples are given in Section 3.

The following quantity plays a key role in this article:

$$
g(A)=\left[N_{2}^{2}(A)-\sum_{k=1}^{n}\left|\lambda_{k}\right|^{2}\right]^{1 / 2}
$$

where $N_{2}(A)=\left(\text { Trace } A A^{*}\right)^{1 / 2}$ is the Frobenius (Hilbert-Schmidt norm) of $A$. Here, $A^{*}$ is adjoint to $A$. The following relations are checked in [7, Section 2.1]:

$$
g^{2}(A) \leq N_{2}^{2}(A)-\mid \text { Trace } A^{2} \mid \text { and } g^{2}(A) \leq \frac{N_{2}^{2}\left(A-A^{*}\right)}{2}=2 N_{2}^{2}\left(A_{I}\right),
$$

where $A_{I}=\left(A-A^{*}\right) / 2 i$. If $A$ is a normal matrix: $A A^{*}=A^{*} A$, then $g(A)=0$.

By $\operatorname{co}(A)$ we denote the closed convex hull of $\sigma(A)$. Let $f(z, w)$ be regular on a neighborhood of $\operatorname{co}(A) \times \operatorname{co}(\tilde{A})$. Put

$$
f^{(j, k)}(z, w)=\frac{\partial^{j+k} f(z, w)}{\partial z^{j} \partial w^{k}},
$$

and let the numbers $\eta_{j k}=\eta_{j k}(f, A, \tilde{A})$ be given by

$$
\begin{gathered}
\eta_{00}=\sup _{z \in \sigma(A), w \in \sigma(\tilde{A})}|f(z, w)| ; \eta_{j k}=\frac{1}{(j ! k !)^{3 / 2}} \sup _{z \in c o(A), w \in \operatorname{co}(\tilde{A})}\left|f^{(j, k)}(z, w)\right| ; \\
\eta_{0 j}:=\frac{1}{(j !)^{3 / 2}} \sup _{z \in \sigma(A), w \in \operatorname{co}(\tilde{A})}\left|\frac{\partial^{j} f(z, w)}{\partial w^{j}}\right|
\end{gathered}
$$

and

$$
\eta_{j 0}:=\frac{1}{(j !)^{3 / 2}} \sup _{z \in c o(A), w \in \sigma(\tilde{A})}\left|\frac{\partial^{j} f(z, w)}{\partial z^{j}}\right| \quad(j, k \geq 1) .
$$

Now we are in a position to formulate the main result of the paper. 
THEOREM 1.1. Let both $A$ and $\tilde{A}$ be non-normal matrices and $f(z, w)$ be regular on a neighborhood of $\operatorname{co}(A) \times \operatorname{co}(\tilde{A})$. Then

$$
\|F(f, A, K, \tilde{A})\| \leq N_{2}(K) \sum_{j, k=0}^{n-1} \eta_{j k} g^{j}(A) g^{k}(\tilde{A}) .
$$

If $A$ is normal, $\tilde{A}$ is non-normal and $f(z, w)$ is regular on a neighborhood of $\sigma(A) \times$ $\operatorname{co}(\tilde{A})$, then

$$
\|F(f, A, K, \tilde{A})\| \leq N_{2}(K) \sum_{j=0}^{n-1} \eta_{0 j} g^{j}(\tilde{A}) .
$$

If $\tilde{A}$ is normal, $A$ is non-normal and $f(z, w)$ is regular on a neighborhood of $\sigma(\tilde{A}) \times$ $\operatorname{co}(A)$, then

$$
\|F(f, A, K, \tilde{A})\| \leq N_{2}(K) \sum_{j=0}^{n-1} \eta_{j 0} g^{j}(A) .
$$

If both $A$ and $\tilde{A}$ are normal and $f(z, w)$ is regular on a neighborhood of $\sigma(A) \times \sigma(\tilde{A})$, then

$$
\|F(f, A, K, \tilde{A})\| \leq N_{2}(K) \max _{j, k}\left|f\left(\lambda_{j}, \tilde{\lambda}_{k}\right)\right|
$$

2. Proof of Theorem 1.1. We need the following result proved in [8].

Lemma 2.1. Let $\Omega$ and $\tilde{\Omega}$ be the closed convex hulls of the complex points $x_{0}, x_{1}, \ldots, x_{n}$ and $y_{0}, y_{1}, \ldots, y_{m}$, respectively, and let a scalar-valued function $f(z, w)$ be regular on a neighborhood of $\Omega \times \tilde{\Omega}$. Additionally, let $L$ and $\tilde{L}$ be the boundaries of $\Omega$ and $\tilde{\Omega}$, respectively. Then with the notation

$$
Y\left(x_{0}, \ldots, x_{n} ; y_{0}, \ldots, y_{m}\right)=-\frac{1}{4 \pi^{2}} \int_{L} \int_{\tilde{L}} \frac{f(z, w) d z d w}{\left(z-x_{0}\right) \cdots\left(z-x_{n}\right)\left(w-y_{0}\right) \cdots\left(w-y_{m}\right)},
$$

we have

$$
\left|Y\left(x_{0}, \ldots, x_{n} ; y_{0}, \ldots, y_{m}\right)\right| \leq \frac{1}{n ! m !} \sup _{z \in \Omega, w \in \tilde{\Omega}}\left|f^{(n, m)}(z, w)\right| .
$$

Let $\left\{e_{k}\right\}$ and $\left\{\tilde{e}_{k}\right\}$ be the orthogonal normal bases of the triangular representation (Schur's bases) to $A$ and $\tilde{A}$, respectively. So,

$$
A e_{k}=\sum_{j=1}^{k} a_{j k} e_{j}
$$


We can write

$$
A=D_{A}+V_{A}, \quad \tilde{A}=D_{\tilde{A}}+V_{\tilde{A}},
$$

where $D_{A}, D_{\tilde{A}}$ are the diagonal parts, $V_{A}$ and $V_{\tilde{A}}$ are the nilpotent parts of $A$ and $\tilde{A}$, respectively. Namely,

$$
D_{A} e_{k}=\lambda_{k} e_{k} ; \quad V_{A} e_{k}=\sum_{j=1}^{k-1} a_{j k} e_{j}
$$

Similarly, $D_{\tilde{A}}$ and $V_{\tilde{A}}$ are defined. Furthermore, let $\left|V_{A}\right|$ be the operator whose entries in $\left\{e_{k}\right\}$ are the absolute values of the entries of a matrix $V_{A}$. That is, $\left(\left|V_{A}\right| e_{j}, e_{k}\right)=$ $\left|\left(V_{A} e_{j}, e_{k}\right)\right|$ and

$$
\left|V_{A}\right|=\sum_{k=1}^{n} \sum_{j=1}^{k-1}\left|a_{j k}\right|\left(\cdot, e_{k}\right) e_{j}
$$

Similarly, $\left|V_{\tilde{A}}\right|$ is defined with respect to $\left\{\tilde{e}_{k}\right\}$. In addition, $|K|$ is defined by

$$
|K| \tilde{e}_{j}=\sum_{k=1}^{n}\left|\left(K \tilde{e}_{j}, e_{k}\right)\right| e_{k} .
$$

LEMMA 2.2. Under the hypothesis of Theorem 1.1, the inequality

$$
\|F(f, A, K, \tilde{A})\| \leq\||K|\| \sum_{j, k=1}^{n-1} \sqrt{k ! j !} \eta_{j k}\left\|\left|V_{\tilde{A}}\right|^{j}\right\|\left\|\left|V_{\tilde{A}}\right|^{k}\right\|
$$

is true, where $V_{A}$ and $V_{\tilde{A}}$ are the nilpotent parts of $A$ and $\tilde{A}$, respectively.

Proof. It is not hard to see that the representation (2.1) implies the equality

$$
(A-I \lambda)^{-1}=\left(D_{A}+V_{A}-\lambda I\right)^{-1}=\left(I+R_{\lambda}\left(D_{A}\right) V_{A}\right)^{-1} R_{\lambda}\left(D_{A}\right)
$$

for all regular $\lambda$. According to Lemma 1.7 .1 from [7] $R_{\lambda}\left(D_{A}\right) V_{A}$ is a nilpotent operator, because $V_{A}$ and $R_{\lambda}\left(D_{A}\right)$ the same invariant subspaces. Hence, $\left(R_{\lambda}\left(D_{A}\right) V_{A}\right)^{n}=0$. Therefore, from (1.1) it follows

$$
F(f, A, K, \tilde{A})=\sum_{j, k=0}^{n-1} M_{j k}
$$

where

$M_{j k}=\frac{(-1)^{k+j}}{4 \pi^{2}} \int_{C_{\tilde{A}}} \int_{C_{A}} f(z, w)\left(R_{z}\left(D_{A}\right) V_{A}\right)^{j} R_{z}\left(D_{A}\right) K\left(R_{w}\left(D_{\tilde{A}}\right) V_{\tilde{A}}\right)^{k} R_{w}\left(D_{\tilde{A}}\right) d z d w$. 
Since $D_{A}$ is a diagonal matrix with respect to the Schur basis $\left\{e_{k}\right\}$ and its diagonal entries are the eigenvalues of $A$, we obtain

$$
R_{z}\left(D_{A}\right)=\sum_{j=1}^{n} \frac{Q_{j}}{\lambda_{j}(A)-z}
$$

where $Q_{k}=\left(\cdot, e_{k}\right) e_{k}$. Similarly,

$$
R_{z}\left(D_{\tilde{A}}\right)=\sum_{j=1}^{n} \frac{\tilde{Q}_{j}}{\lambda_{j}(\tilde{A})-z},
$$

where $\tilde{Q}_{k}=\left(\cdot, \tilde{e}_{k}\right) \tilde{e}_{k}$. Taking into account that $Q_{s} V_{A} Q_{m}=0, \tilde{Q}_{s} V_{\tilde{A}} \tilde{Q}_{m}=0(s \geq m)$, we get

$$
\begin{gathered}
M_{j k}=\sum_{1 \leq s_{1}<s_{2}<\cdots<s_{j+1} \leq n} Q_{s_{1}} V_{A} Q_{s_{2}} V_{A} \cdots V_{A} Q_{s_{j+1}} K \times \\
\times \sum_{1 \leq m_{1}<m_{2}<\cdots<m_{k+1} \leq n} \tilde{Q}_{m_{1}} V_{\tilde{A}} \tilde{Q}_{m_{2}} V_{\tilde{A}} \cdots V_{\tilde{A}} \tilde{Q}_{m_{k+1}} \hat{I}\left(s_{1}, \ldots, s_{j+1}, m_{1}, \ldots, m_{k+1}\right),
\end{gathered}
$$

where $0 \leq j, k \leq n-1$ and

$$
\begin{gathered}
\hat{I}\left(s_{1}, \ldots, s_{j+1}, m_{1}, \ldots m_{k+1}\right)= \\
\frac{(-1)^{k+j}}{4 \pi^{2}} \int_{C_{A}} \int_{C_{\tilde{A}}} \frac{f(z, w) d z d w}{\left(\lambda_{s_{1}}(A)-z\right) \cdots\left(\lambda_{s_{k+1}}(A)-z\right)\left(\lambda_{m_{1}}(\tilde{A})-w\right) \cdots\left(\lambda_{m_{k+1}}(\tilde{A})-w\right)} .
\end{gathered}
$$

Hence, with $M_{j k}=M$, we have

$$
\begin{aligned}
&\left|\left(M \tilde{e}_{m}, e_{s}\right)\right|=\mid \sum_{s<s_{2}<\cdots<s_{j+1} \leq n} \sum_{1 \leq m_{1}<m_{2}<\cdots<m} \hat{I}\left(s, \ldots, s_{j+1}, m_{1}, \ldots, m\right) \times \\
& \times\left(Q_{s} V_{A} Q_{s_{2}} V_{A} \cdots V_{A} Q_{s_{j+1}} K \tilde{Q}_{m_{1}} V_{\tilde{A}} \tilde{Q}_{m_{2}} V_{\tilde{A}} \cdots V_{\tilde{A}} \tilde{Q}_{m} \tilde{e}_{m}, e_{s}\right) \mid \leq J_{j k} \sum_{s<s_{2}<\cdots<s_{j+1} \leq n} \\
& \times \sum_{1 \leq m_{1}<m_{2}<\cdots<m}\left(Q_{s}\left|V_{A}\right| Q_{s_{2}}\left|V_{A}\right| \cdots Q_{s_{j+1}}|K| \tilde{Q}_{m_{1}}\left|V_{\tilde{A}}\right| \tilde{Q}_{m_{2}}\left|V_{\tilde{A}}\right| \cdots \tilde{Q}_{m} \tilde{e}_{m}, e_{s}\right),
\end{aligned}
$$

where

$$
J_{j k}:=\max _{1 \leq s_{1}<\cdots<s_{j+1} \leq n ; 1 \leq m_{1}<\cdots<m_{k+1} \leq n}\left|\hat{I}\left(s_{1}, \ldots, s_{j+1}, m_{1}, \ldots m_{k+1}\right)\right| .
$$


Thus $\left|\left(M \tilde{e}_{m}, e_{s}\right)\right| \leq\left(T \tilde{e}_{m}, e_{s}\right)$, where

$$
\begin{gathered}
T=J_{j k} \sum_{s_{1}<s_{2}<\cdots<s_{j+1} \leq n} \sum_{1 \leq m_{1}<m_{2}<\cdots<m_{k+1} \leq n} Q_{s_{1}}\left|V_{A}\right| Q_{s_{2}}\left|V_{A}\right| \cdots\left|V_{A}\right| Q_{s_{j+1}}|K| \times \\
\times \tilde{Q}_{m_{1}}\left|V_{\tilde{A}}\right| \tilde{Q}_{m_{2}}\left|V_{\tilde{A}}\right| \cdots\left|V_{\tilde{A}}\right| \tilde{Q}_{m_{k+1}} .
\end{gathered}
$$

Take into account that

$$
M x=\sum_{k=1}^{n}\left(x, \tilde{e}_{k}\right) M \tilde{e}_{k}=\sum_{j=1}^{n} \sum_{k=1}^{n}\left(x, \tilde{e}_{k}\right)\left(M \tilde{e}_{k}, e_{j}\right) e_{j} \quad\left(x \in \mathbb{C}^{n}\right) .
$$

So

$$
\begin{gathered}
\|M x\|^{2}=\sum_{j=1}^{n}\left|\sum_{k=1}^{n}\left(x, \tilde{e}_{k}\right)\left(M \tilde{e}_{k}, e_{j}\right)\right|^{2} \leq \\
\sum_{j=1}^{n}\left(\sum_{k=1}^{n}\left(x, \tilde{e}_{k}\right)\left(T \tilde{e}_{k}, e_{j}\right)\right)^{2} \cdot
\end{gathered}
$$

Since $\|x\|=\|y\|$ for

$$
y=\sum_{k=1}^{n}\left|\left(x, \tilde{e}_{k}\right)\right| \tilde{e}_{k},
$$

we obtain $\|M\| \leq\|T\|$. But

$$
\sum_{1 \leq s_{1}<s_{2}<\cdots<s_{j+1} \leq n} Q_{s_{1}}\left|V_{A}\right| Q_{s_{2}}\left|V_{A}\right| \cdots\left|V_{A}\right| Q_{s_{j+1}}=\left|V_{A}\right|^{j}
$$

and

$$
\sum_{1 \leq m_{1}<m_{2}<\cdots<m_{k+1} \leq n} \tilde{Q}_{m_{1}}\left|V_{\tilde{A}}\right| \tilde{Q}_{m_{2}}\left|V_{\tilde{A}}\right| \cdots\left|V_{\tilde{A}}\right| Q_{m_{k+1}}=\left|V_{\tilde{A}}\right|^{k} .
$$

So by (2.3)

$$
\left\|M_{j k}\right\| \leq\|T\| \leq J_{j k}\left\|\left|V_{A}\right|^{j}|K|\left|V_{\tilde{A}}\right|^{k}\right\|(j, k \geq 0) .
$$

Due to Lemma 2.1

$$
J_{j k} \leq \sup _{z \in c o(A), w \in \operatorname{co}(\tilde{A})} \frac{\left|f^{(j, k)}(z, w)\right|}{j ! k !}=\sqrt{j ! k !} \eta_{j k} \quad(j, k \geq 1) .
$$


Thus,

$$
\left\|M_{j k}\right\| \leq \sqrt{j ! k !} \eta_{j k}\left\|\left|V_{A}\right|^{j}|K|\left|V_{\tilde{A}}\right|^{k}\right\|(j, k \geq 0)
$$

This inequality and (2.2) imply the required result. $\square$

Proof of Theorem 1.1. Theorem 2.5.1 from [7] implies

$$
\left\|W^{k}\right\| \leq \frac{1}{\sqrt{k !}} N_{2}^{k}(W)
$$

for any $n \times n$ nilpotent matrix $W$. Take into account that $N_{2}\left(\left|V_{A}\right|\right)=N_{2}\left(V_{A}\right)$. Moreover, by Lemma 2.3.2 from [7], $N_{2}\left(V_{A}\right)=g(A)$. Thus,

$$
\left\|\left|V_{A}\right|^{k}\right\| \leq \frac{1}{\sqrt{k !}} g^{k}(A) \quad(k=1, \ldots, n-1) .
$$

The similar inequality holds for $V_{\tilde{A}}$. In addition,

$$
N_{2}^{2}(|K|)=\sum_{j=1}^{n}\left\||K| \tilde{e}_{j}\right\|^{2}=\sum_{j=1}^{n} \sum_{k=1}^{n}\left|\left(K \tilde{e}_{j}, e_{k}\right)\right|^{2}=\sum_{j=1}^{n} \sum_{k=1}^{n}\left\|K \tilde{e}_{j}\right\|^{2}=N_{2}^{2}(K)
$$

Now the previous lemma yields the required result. [

3. Examples. Consider the equation

$$
A X-X \tilde{A}=K
$$

assuming that

$$
\delta:=\operatorname{dist}(\operatorname{co}(A), \operatorname{co}(\tilde{A}))>0 .
$$

Take $f(z, w)=\frac{1}{z-w}$. Then

$$
\eta_{j k} \leq \frac{(k+j) !}{\delta^{j+k+1}(k ! j !)^{3 / 2}} \quad(j, k=0,1, \ldots, n-1) .
$$

Hence, by Theorem 1.1 and (1.3) a solution of (3.1) satisfies the inequality

$$
\|X\| \leq N_{2}(K) \sum_{j, k=0}^{n-1} \frac{(k+j) !}{\delta^{j+k+1}(k ! j !)^{3 / 2}} g^{j}(A) g^{k}(\tilde{A}) .
$$

Finally, consider the function

$$
S(x):=-\frac{1}{4 \pi^{2}} \int_{C_{\tilde{A}}} \int_{C_{A}} \sin (x(z+w)) R_{z}(A) K R_{w}(\tilde{A}) d w d z \quad(x \in \mathbb{R}) .
$$


We have

$$
S^{\prime \prime}(x)=\frac{1}{4 \pi^{2}} \int_{C_{\tilde{A}}} \int_{C_{A}}(z+w)^{2} \sin (x(z+w)) R_{z}(A) K R_{w}(\tilde{A}) d w d z .
$$

But $z R_{z}(A)=A R_{z}(A)-I$ and therefore,

$$
z^{2} R_{z}(A)=z A R_{z}(A)-z I=A\left(A R_{z}(A)-I\right)-z I=A^{2} R_{z}(A)-I-z I .
$$

So, $S(x)$ is a solution of the equation

$$
S^{\prime \prime}=A^{2} S+A S \tilde{A}+S \tilde{A}^{2} .
$$

\section{REFERENCES}

[1] R. Arens and A.P. Calderon. Analytic functions of several Banach algebra elements. Ann. Math., 62:204-216, 1955.

[2] Yu.L. Daleckii and M.G. Krein. Stability of Solutions of Differential Equations in Banach Space. Translations of Mathematical Monographs, Vol. 43, Amer. Math. Soc., Providence, R.I., 1974.

[3] M. Dehghan and M. Hajarian. The reflexive and anti-reflexive solutions of a linear matrix equation and systems of matrix equations. Rocky Mountain J. Math., 40(3):825-848, 2010.

[4] F.R. Gantmacher. The Matrix Theory (in Russian). Nauka, Moscow, 1967.

[5] I.M. Gel'fand and G.E. Shilov. Some Questions of Theory of Differential Equations (in Russian). Nauka, Moscow, 1958.

[6] M.I. Gil'. Estimates for norm of matrix-valued functions. Linear Multilinear Algebra, 35:65-73, 1993.

[7] M.I. Gil'. Operator Functions and Localization of Spectra. Lecture Notes in Mathematics, Vol. 1830, Springer-Verlag, Berlin, 2003.

[8] M.I. Gil'. Norms of functions of commuting matrices. Electron. J. Linear Algebra, 13:122-130, 2005.

[9] M.I. Gil'. Difference Equations in Normed Spaces. Stability and Oscillations, NorthHolland Mathematics Studies, Vol. 206, Elsevier, Amsterdam, 2007.

[10] R.A. Horn and C.R. Johnson. Topics in Matrix Analysis. Cambridge University Press, Cambridge, 1991.

[11] K. Jbilou. ADI preconditioned Krylov methods for large Lyapunov matrix equations. Linear Algebra Appl., 432(10):2473-2485, 2010.

[12] M. Konstantinov, D.-W. Gu, V. Mehrmann, and P. Petkov. Perturbation Theory for Matrix Equations. Studies in Computational Mathematics, Vol. 9, North-Holland Publishing Co., Amsterdam, 2003.

[13] Y. Liu and Y. Tian. How to use RSVD to solve the matrix equation $A=B X C^{\prime} A=$ BXC. Linear Multilinear Algebra, 58:537-543, 2010.

[14] A.G. Mazko. Matrix Equations, Spectral Problems and Stability of Dynamic Systems. Stability, Oscillations and Optimization of Systems, Scientific Publishers, Cambridge, 2008.

[15] V. Müller. Spectral Theory of Linear Operators. Birkhäusr Verlag, Basel, 2003.

[16] J.L. Taylor. Analytic functional calculus for several commuting operators. Acta Math., 125:1-38, 1970.

[17] B. Zhou, J. Lam, and G.-R. Duan. On Smith-type iterative algorithms for the Stein matrix equation. Appl. Math. Lett., 22(7):1038-1044, 2009. 